\title{
Physics of the intermediate layer between a plasma and a collisionless sheath and mathematical meaning of the Bohm criterion
}

Cite as: Phys. Plasmas 19, 073514 (2012); https://doi.org/10.1063/1.4737080

Submitted: 09 May 2012 . Accepted: 11 June 2012. Published Online: 20 July 2012

N. A. Almeida, and M. S. Benilov

\section{ARTICLES YOU MAY BE INTERESTED IN}

Plasma-sheath transition in the kinetic Tonks-Langmuir model

Physics of Plasmas 13, 063508 (2006); https://doi.org/10.1063/1.2209928

The influence of collisions on the plasma sheath transition

Physics of Plasmas 4, 4158 (1997); https://doi.org/10.1063/1.872536

On the consistency of the collisionless sheath model

Physics of Plasmas 9, 4427 (2002); https://doi.org/10.1063/1.1513155

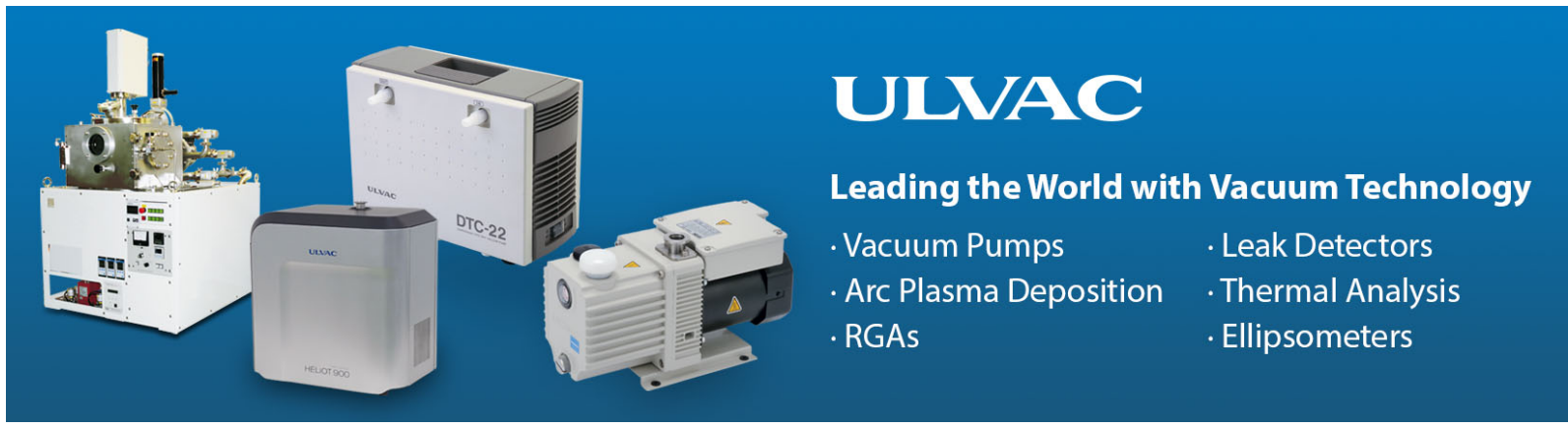




\title{
Physics of the intermediate layer between a plasma and a collisionless sheath and mathematical meaning of the Bohm criterion
}

\author{
N. A. Almeida and M. S. Benilov \\ Departamento de Física, CCCEE, Universidade da Madeira Largo do Município, 9000 Funchal, Portugal
}

(Received 9 May 2012; accepted 11 June 2012; published online 20 July 2012)

\begin{abstract}
A transformation of the ion momentum equation simplifies a mathematical description of the transition layer between a quasi-neutral plasma and a collisionless sheath and clearly reveals the physics involved. Balance of forces acting on the ion fluid is delicate in the vicinity of the sonic point and weak effects come into play. For this reason, the passage of the ion fluid through the sonic point, which occurs in the transition layer, is governed not only by inertia and electrostatic force but also by space charge and ion-atom collisions and/or ionization. Occurrence of different scenarios of asymptotic matching in the plasma-sheath transition is analyzed by means of simple mathematical examples, asymptotic estimates, and numerical calculations. In the case of a collisionless sheath, the ion speed distribution plotted on the logarithmic scale reveals a plateau in the intermediate region between the sheath and the presheath. The value corresponding to this plateau has the meaning of speed with which ions leave the presheath and enter the sheath; the Bohm speed. The plateau is pronounced reasonably well provided that the ratio of the Debye length to the ion mean free path is of the order of $10^{-3}$ or smaller. There is no such plateau if the sheath is collisional and hence no sense in talking of a speed with which ions enter the sheath. (C) 2012 American Institute of Physics. [http://dx.doi.org/10.1063/1.4737080]
\end{abstract}

\section{INTRODUCTION}

The topic of plasma-sheath transition near a negative surface is of central importance for the theory and modelling of bounded plasmas. There has been considerable interest in the literature during the last decades towards different aspects of the plasma-sheath transition, including the Bohm criterion; see, e.g., reviews. ${ }^{1-5}$

The concepts of a quasi-neutral plasma and a spacecharge sheath are only meaningful if the characteristic Debye length $\lambda_{D}$ is small. Therefore, elucidating features of the plasma-sheath transition originating in the inequality $\lambda_{D} \ll L$, where $L$ is a length scale characterizing the presheath, i.e., a quasi-neutral plasma region adjacent to the sheath, is necessary for understanding this transition. Consequently, an appropriate means for investigation of plasmasheath transition is an asymptotic approach treating $\lambda_{D} / L$ as a small parameter and employing the method of matched asymptotic expansions (e.g., Refs. 6-11), which is a standard tool for solving multi-scale problems and represents a powerful alternative to intuitive approaches.

In the case of a collisionless sheath, the method of matched asymptotic expansions was applied to the plasmasheath transition in Ref. 12; a refined analysis was given in Ref. 13. Since then, many works have been published on asymptotic treatment of different aspects of this transition, e.g., Refs. 2-5. The asymptotic structure of the plasmasheath transition includes three zones, each described by a separate asymptotic expansion: a quasi-neutral plasma, a space-charge sheath, and an intermediate transition layer. (Note that the latter was termed the first transitional layer in Ref. 12, the transonic layer in Ref. 14, and the intermediate region in Ref. 13.) The asymptotic expansions describing the plasma and the sheath have a clear physical meaning and represent an adequate mathematical description of the wellknown physical concepts going back to Langmuir. On the contrary, the transition layer has appeared in the course of analyses $^{12,13}$ as a purely mathematical concept: a direct matching of the plasma and sheath expansions is impossible beyond the first approximation.

The topic of transition layer has been revisited in subsequent works, e.g., Refs. 5, 15-17, however a clear physical interpretation of the asymptotic solution is still lacking. This renders the theory of plasma-sheath transition not quite complete and has contributed to a number of controversies. Some researchers even believe that the transition layer is not distinguished by special physical processes but rather represents a mathematical tool to bridge the sheath edge singularity, which amounts to the transition layer being little more than an artefact produced by the method of matched asymptotic expansions.

In this work, the question of physics of the transition layer is reconsidered on the following grounds. The ion velocity $v_{i}$, ion and electron densities $n_{i}$ and $n_{e}$, and potential $\varphi$ remain to the first approximation constant in the transition layer. Therefore, the transition layer must be described by means of the second approximation, i.e., by two-term asymptotic expansions: the expansion of each of the quantities $v_{i}, n_{i}, n_{e}$, and $\varphi$ includes a constant first-order term and a second-order term describing the variation of this quantity in the transition layer. Unfortunately, equations governing these variations cannot be derived in a straightforward way by means of considering only the second approximation, so three-term expansions have been used. ${ }^{13}$ [Derivations given in other works which do not employ formal asymptotic expansions involve approximations similar to using 
three-term expansions: derivation ${ }^{15}$ relies on equations comprising terms of both second and third order (Eqs. (29) and (30)); derivations ${ }^{12,16,17}$ use three-term expansions in powers of potential of the ion and electron densities in the Poisson equation.] It is the use of three-term expansions for derivation of equations governing the second term that obscures the physics. However, the necessity to use threeterm expansions is not an inherent feature of the considered problem and may be eliminated by properly transforming the ion momentum equation. ${ }^{18}$ One can hope that such approach will distinctly reveal the physics involved, as the method of matched asymptotic expansions always does.

Another question considered in this work is as follows. There are many papers employing different definitions of a collisionally modified Bohm criterion, ${ }^{19}$ being apparently the first and most cited example and ${ }^{20-25}$ the most recent ones. It is striking that none of these definitions has gained wide recognition. This can only be explained by all these definitions being arbitrary. Then a question arises: If there is a unique Bohm criterion for a collisionless sheath, why cannot there be a unique Bohm criterion for collisional sheaths? One can mention in this connection the work, ${ }^{22}$ which was aimed at a nonarbitrary definition of a collisionally modified Bohm criterion, and the subsequent discussion; ${ }^{26,27}$ other relevant references are works, ${ }^{15,28}$ which are specifically concerned with the effect of collisions on the plasma-sheath transition. One of the factors feeding the confusion is the uncertainty with identification of the Bohm criterion in results of numerical solution of a full problem, i.e., of a problem which comprises the Poisson equation and therefore does not involve an a priori sub-division of the calculation domain into the quasi-neutral plasma and a space-charge sheath; for example, the conclusion of Ref. 22 was that there are no peculiarities at the Bohm speed in distributions of parameters, including in cases where the sheath is collisionless.

Of course, the bottom question is: What is the mathematical meaning of the Bohm criterion? A frequently encountered idea is that the Bohm criterion is related to the sheath edge singularity. A different point of view is suggested by the character of asymptotic solutions: ${ }^{12,13}$ the Bohm criterion represents a manifestation of one of general scenarios of asymptotic matching, namely, matching on a constant. In this work, this question is considered with the use of simple mathematical examples, asymptotic estimates, and results of numerical solution of a full problem.

The outline of the paper is as follows. Governing equations are briefly introduced in Sec. II. Section III is concerned with elucidating the physics of transition layer between a plasma and a collisionless sheath. The mathematical meaning of the Bohm criterion is analyzed in Sec. IV. Conclusions are summarized in Sec. V. In the Appendix, the structure of the plasma-sheath transition for collisionless to moderately collisional to collision-dominated sheaths is analyzed in terms of simple asymptotic estimates.

\section{EQUATIONS}

Let us consider a transition from a weakly ionized plasma with cold ions to an absorbing surface under a nega- tive potential. The plasma is planar with ion-atom collisions and/or ionization. Governing equations are written in the fluid approximation and are well-known; they include the ion conservation equation written with account of ionization, the ion momentum equation written with account of the friction force due to collisions, the equilibrium equation for the electrons, and the Poisson equation,

$$
\begin{gathered}
\frac{d}{d x}\left(n_{i} v_{i}\right)=k_{i} n_{a} n_{e}, \\
\frac{d}{d x}\left(m_{i} n_{i} v_{i}^{2}\right)=e n_{i} E-n_{i} \nu_{i} m_{i} v_{i}, \\
\frac{d}{d x}\left(n_{e} k T_{e}\right)+e n_{e} E=0, \\
\varepsilon_{0} \frac{d E}{d x}=e\left(n_{i}-n_{e}\right),
\end{gathered}
$$

where $k_{i}$ is the ionization rate coefficient, $\nu_{i}=\nu_{i}\left(\left|v_{i}\right|\right)$ is the frequency of momentum transfer from an ion to neutral atoms, and all the other designations are the usual ones. The $x$-axis is directed from the surface into the plasma, so $v_{i}<0$ and $E<0$.

The above equations may be characterized by a number of length scales: a Debye length $\lambda_{D}$, a characteristic mean free path of ions in the gas of atoms, $\lambda_{s}$, and an ionization length $l_{i}$. A further length scale is the width $\Delta$ of the plasma slab. This work is concerned with the situation where $\lambda_{D} \ll l_{i}, \Delta$, so the discharge gap may be subdivided into the region of quasi-neutral plasma and the sheath, the ionization in the sheath being insignificant. Three cases will be treated: the sheath is collisionless while collisions and/or ionization play a role in the plasma, $\lambda_{D} \ll \min \left(\lambda_{s}, l_{i}\right) \lesssim \Delta$; the sheath is moderately collisional, $\lambda_{D} \sim \lambda_{s} \ll l_{i}, \Delta$; the sheath is collision-dominated, $\lambda_{s} \ll \lambda_{D} \ll l_{i}, \Delta$.

We assume that lengths $\lambda_{D}, \lambda_{s}$, and $l_{i}$ are estimated for conditions characteristic of the sheath. In the case of a collisionless sheath, it is convenient to use more specific definitions: $\lambda_{s}$ and $l_{i}$ are estimated in terms of the Bohm speed, $\lambda_{s}=v_{s} / \nu_{i}\left(v_{s}\right), l_{i}=v_{s} / k_{i} n_{a}$, and $\lambda_{D}$ is estimated in terms of the corresponding charge particle density $n_{s}=j_{i} / e v_{s}$ (here $v_{s}=\sqrt{k T_{e} / m_{i}}$ is the Bohm speed or, equivalently, the speed of ion sound wave and $j_{i}$ is the density of ion current to the surface). It is convenient also to introduce the length $l=\left(\lambda_{s}^{-1}+2 l_{i}^{-1}\right)^{-1}$, which is of the order of the smaller of the lengths $\lambda_{s}$ and $l_{i}$.

\section{PHYSICS OF THE TRANSITION LAYER BETWEEN A PLASMA AND A COLLISIONLESS SHEATH}

\section{A. Transforming ion momentum equation}

An equivalent form of the ion momentum equation (2) can be obtained with the use of Eq. (1),

$$
m_{i} n_{i} v_{i} \frac{d v_{i}}{d x}=e n_{i} E-\left(n_{i} \nu_{i}+k_{i} n_{a} n_{e}\right) m_{i} v_{i}
$$

Another equivalent form of this equation may be derived similarly to the way in which it was done in the study of a 
transition from a collision-dominated plasma to a collisionfree space-charge sheath with account of variable ion temperature. ${ }^{18}$ Eliminating from Eq. (3) $n_{e}$ with the use of Eq. (4) and then eliminating from the resulting relationship $d n_{i} / d x$ with the use of Eq. (1), one can obtain

$$
e n_{i} E=\frac{n_{i} k T_{e}}{v_{i}} \frac{d v_{i}}{d x}+\varepsilon_{0}\left(\frac{k T_{e}}{e} \frac{d^{2} E}{d x^{2}}+E \frac{d E}{d x}\right)-\frac{k T_{e}}{v_{i}} k_{i} n_{a} n_{e} .
$$

Substituting this expression for the electrostatic force term of Eq. (5), one obtains

$$
\begin{aligned}
\frac{v_{i}}{v_{s}^{2}} \frac{d v_{i}}{d x}-\frac{1}{v_{i}} \frac{d v_{i}}{d x}= & \frac{\varepsilon_{0}}{e n_{i}}\left(\frac{d^{2} E}{d x^{2}}+\frac{e E}{k T_{e}} \frac{d E}{d x}\right) \\
& +\frac{1}{v_{s}}\left[-\nu_{i} \frac{v_{i}}{v_{s}}-k_{i} n_{a} \frac{n_{e}}{n_{i}}\left(\frac{v_{i}}{v_{s}}+\frac{v_{s}}{v_{i}}\right)\right] .
\end{aligned}
$$

\section{B. Asymptotic analysis}

A treatment of the considered problem in the case of a collisionless sheath, $\lambda_{D} \ll l$, by means of the method of matched asymptotic expansions in the small parameter $\lambda_{D} / l$ was given in Refs. 12 and 13. The procedure of the method of matched asymptotic expansions includes two steps. First, a reasonable guess should be made of the asymptotic structure of the solution, i.e., relevant asymptotic zones and scalings of unknown variables in these zones. At the second step, the guess is formalized: formal asymptotic expansions are written and differential equations describing each zone are derived and solved. The guess made at the first step is correct if equations in each zone are solvable and solutions in each pair of adjacent zones can be asymptotically matched; otherwise the guess must be reconsidered.

The first step is performed by means of asymptotic estimates. These estimates can be made in different ways, e.g., with the use of considerations stemming from matching as in Ref. 13. Here, asymptotic estimates are performed with the use of Eq. (7). Let us designate by $\delta$ a local scale of variation of parameters and assume that the local electric field $E$ is of the order of $k T_{e} / e \delta, v_{i}$ of the order of $v_{s}$, and $n_{i}$ and $n_{e}$ of the order of $n_{s}$. The lhs of Eq. (7) and the two terms on the rhs are of the order, respectively, $\delta^{-1}, \lambda_{D}^{2} / \delta^{3}, l^{-1}$. The latter estimates suggest two scalings: $\delta=l$ and $\delta=\lambda_{D}$. On the scale $\delta=l$, the first term on the rhs of Eq. (7) is of the order of $\left(\lambda_{D} / l\right)^{2}$ relative to the other terms, i.e., negligible: the quasineutral plasma, i.e., the presheath. On the scale $\delta=\lambda_{D}$, the second term on the rhs of Eq. (7) is of the order of $\lambda_{D} / l$ relative to the other terms, i.e., negligible: the space-charge sheath without collisions and ionization.

Since the density of the space charge near a negative surface is positive and increases in the direction to the surface, $d E / d x>0$ and $d^{2} E / d x^{2}<0$. Hence, the first term on the rhs of Eq. (7) is negative. The second term is obviously positive. It follows that the rhs of Eq. (7) is positive in the presheath, where the second term is dominating, and negative in the sheath, where the first term is dominating. Since the ions are accelerated in the direction to the surface, i.e., $d v_{i} / d x>0$, one concludes that the ion motion is subsonic, $\left|v_{i}\right|<v_{s}$, in the presheath and supersonic, $\left|v_{i}\right|>v_{s}$, in the sheath.

Thus, the second term on the rhs of Eq. (7) is dominating for $\left|v_{i}\right|<v_{s}$ and the first term is dominating for $\left|v_{i}\right|>v_{s}$. Therefore, there should be an intermediate transition layer, i.e., a layer where $\left|v_{i}\right|$ is close to $v_{s}$ and where the two terms on the rhs of Eq. (7) are comparable. Let us represent $\left|v_{i}\right|=v_{s}+u_{i},\left|u_{i}\right| \ll v_{s}$ in this layer. Relative variations of the ion and electron densities in this layer are of the same order as the relative variation of the ion speed, i.e., one can represent $n_{i}=n_{s}+n_{i 1}, n_{e}=n_{s}+n_{e 1}$, where $n_{i 1}$ and $n_{e 1}$ are of the order $n_{s} u_{i} / v_{s}$. Substituting the above two-term expansion into Eq. (3) and dropping small terms, one obtains a simplified form of this equation in the transition layer,

$$
k T_{e} \frac{d n_{e 1}}{d x}+e n_{s} E=0
$$

and it follows that $E$ is of the order of $\frac{u_{i}}{v_{s}} \frac{k T_{e}}{e \delta}$. The simplified form of Eq. (7) is

$$
\frac{2 u_{i}}{v_{s}^{2}} \frac{d u_{i}}{d x}=\frac{\varepsilon_{0}}{e n_{s}} \frac{d^{2} E}{d x^{2}}+\frac{1}{l} .
$$

The term on the lhs of Eq. (9) is of the order $u_{i}^{2} / v_{s}^{2} \delta$, the terms on the rhs are of the orders of $\frac{u_{i}}{v_{s}} \frac{\lambda_{D}^{2}}{\delta^{3}}$ and $1 / l$, respectively. Assuming that all the three terms are comparable, one finds that $\delta=\lambda_{D}^{4 / 5} l^{1 / 5}$ and $u_{i} / v_{s}$ is of the order $\left(\lambda_{D} / l\right)^{2 / 5}$. The electric field is of the order of $k T_{e} / e \lambda_{D}^{2 / 5} l^{3 / 5}$.

It should be stressed that each of the terms on the lhs of Eq. (7) is of the order of $\lambda_{D}^{-2 / 5} l^{-3 / 5}$ in the transition layer. The terms on the rhs of Eq. (7) are of the order of $1 / l$, i.e., much smaller. However, the terms on the lhs virtually cancel and their difference is of the order of $1 / l$, and that is why the terms on the rhs of Eq. (7) are retained, albeit in a simplified form, in Eq. (9). It is because of this cancellation that the term on the lhs of Eq. (9) is nonlinear; a situation not typical for approximations of the second and subsequent orders in the perturbation theory.

The simplified form of Eqs. (1), (2), and (4) in the transition layer is

$$
\begin{aligned}
& v_{s} \frac{d n_{i 1}}{d x}+n_{s} \frac{d u_{i}}{d x}=0, \quad m_{i} v_{s}\left(v_{s} \frac{d n_{i 1}}{d x}+2 n_{s} \frac{d u_{i}}{d x}\right)=e n_{s} E \\
& n_{i 1}=n_{e 1} .
\end{aligned}
$$

With the use of Eqs. (10), one can eliminate $E$ from Eq. (9) and obtain an equation involving only $u_{i}$,

$$
\frac{2 u_{i}}{v_{s}^{2}} \frac{d u_{i}}{d x}=\frac{\lambda_{D}^{2}}{v_{s}} \frac{d^{3} u_{i}}{d x^{3}}+\frac{1}{l} .
$$

For the following, we need the asymptotic behavior of function $u_{i}(x)$ for small and large $x / \delta$. Assuming that $u_{i}$ decreases from positive values for small $x / \delta$ to negative values for large $x / \delta$, one readily finds from Eq. (11) that $u_{i} \approx 6 v_{s}\left(\lambda_{D} / x\right)^{2}$ and $u_{i} \approx-v_{s} \sqrt{x / l}$, respectively. 


\section{Discussion}

The asymptotic structure derived above with the use of Eq. (7) is, of course, the same as that of works, ${ }^{12,13}$ and so is Eq. (11). There is, however, the following methodological difference. Equations (8) and (10), which represent the simplified form of the original equations (1)-(4) in the transition layer, are linearly dependent. Hence, one more equation is needed. The lacking equation, Eq. (11), was derived in Ref. 13 with the use of a three-term expansion. In the above analysis, Eq. (11) was derived from Eq. (7) without resorting to a three-term expansion.

In addition to simplifying the analysis, Eq. (7) clearly reveals the physics of the transition layer. The ion fluid is accelerated by the electrostatic force and is retarded by the friction force originating in elastic collisions ion-atom and/or ionization; cf. Eq. (5). The electrostatic force may be represented as the sum of three components as shown by Eq. (6). Two of the components are associated with, respectively, deviations from quasi-neutrality and the ionization; the second and third terms on the rhs of Eq. (6). The other component is present even if the plasma is quasi-neutral and the ionization frozen; the first term on the rhs of Eq. (6).

The first term on the lhs of Eq. (7) represents the ion inertia force; cf. the lhs of Eq. (5). The second term on the lhs of Eq. (7) represents the quasi-neutral no-ionization component of the electrostatic force. The first term on the rhs represents the component of the electrostatic force associated with space charge. The second term on the rhs represents the ionization-associated component of the electrostatic force combined with the friction force. In the presheath, the second term on the rhs of Eq. (7) is dominating, i.e., the spacecharge component of the electrostatic force is insignificant compared to the ionization component and the friction force. It is the other way round in the sheath. In the transition layer, the terms on the rhs of Eq. (7) are of the same order, i.e., the space-charge and ionization components of the electrostatic force and the friction force are comparable.

It should be emphasized that the terms on the rhs of Eq. (7) in the transition layer are much smaller than each term on the lhs. In other words, the inertia and the electrostatic force are dominating in the transition layer and the main contribution to the electrostatic force is given by the quasi-neutral no-ionization component. However, the inertia force and the quasi-neutral no-ionization component of the electrostatic force virtually cancel and their difference is of the same order that the space-charge and ionization components of the electrostatic force and the friction force. For this reason, Eq. (9), which represents the limiting form of Eq. (7) in the transition layer, accounts not only for the inertia force and the quasi-neutral no-ionization component of the electrostatic force but also for separation of charges and ion-atom collisions and/or ionization.

In other words, the transition layer, which is where the passage of the ion fluid through the sonic point $\left|v_{i}\right|=v_{s}$ occurs, is positioned in-between the plasma and the sheath and ion-atom collisions and/or ionization are no longer significant here while separation of charges is not significant yet. However, the balance of forces acting over the ion fluid is delicate in the vicinity of the sonic point and the abovementioned weak effects (ion-atom collisions and/or ionization and separation of charges) also play a role.

In view of the above, it seems that the most adequate term for this layer is the "transonic layer," suggested in Ref. 14.

\section{MATHEMATICAL MEANING OF THE BOHM CRITERION}

The analysis of Sec. III applies to the case of a collisionless sheath without ionization, the aim being to elucidate the physical meaning of the second approximation describing the transition layer. This section is concerned with a comparative analysis of the character of the first approximation in a more general case of a collisionless to moderately collisional to collision-dominated sheath.

\section{A. Mathematical examples}

Let us consider simple mathematical examples illustrating scenarios of asymptotic matching relevant for plasmasheath transition. The first example is the function,

$$
w(\xi)=\frac{1+\varepsilon}{1+\varepsilon+\xi}+2 \exp \left(-\frac{\xi}{\varepsilon}\right),
$$

where the independent variable $\xi$ varies in the domain $\xi \geq 0$ and $\varepsilon$ is a small parameter. This function is plotted in Figure 1(a) for several values of $\varepsilon$. For each $\varepsilon$, there are two regions of variation of function $w(\xi): \xi$ of the order unity and $\xi$ of the order $\varepsilon$; the so-called outer region and the inner region or boundary layer. Approximate expressions describing function $w(\xi)$ in the outer and inner regions are, respectively,

$$
w^{(o)}(\xi)=\frac{1}{1+\xi}, \quad w^{(i)}(\eta)=1+2 e^{-\eta},
$$

where $\eta=\xi / \varepsilon$. The first expression is obtained from Eq. (12) by setting $\varepsilon \rightarrow 0$, the second one is obtained by eliminating from Eq. (12) $\xi$ in terms of $\eta$ and then setting $\varepsilon \rightarrow 0$.

The outer and inner approximations must coincide in the intermediate region $\varepsilon \ll \xi \ll 1$; the so-called asymptotic matching. Indeed, setting $\xi \ll 1$ in the first expression in Eq. (13) and $\eta \gg 1$ in the second expression, one finds $w^{(o)} \approx w^{(i)} \approx 1$.

As the second example, let us consider the function

$$
w(\xi)=\frac{1+\xi}{\varepsilon+\xi}+\frac{1}{\varepsilon} \exp \left(-\frac{\xi}{\varepsilon}\right) .
$$

This function is plotted in Figure 1(b). The outer and inner approximations in this example are, respectively,

$$
w^{(o)}(\xi)=1+\frac{1}{\xi}, \quad w^{(i)}(\eta)=\frac{1}{\varepsilon}\left(\frac{1}{1+\eta}+e^{-\eta}\right) .
$$

In the intermediate region $\varepsilon \ll \xi \ll 1$ the outer and inner approximations read $w^{(o)} \approx 1 / \xi, w^{(i)} \approx 1 / \varepsilon \eta$ and coincide as they should, although they are not constant, in contrast to the preceding example. 
(a)

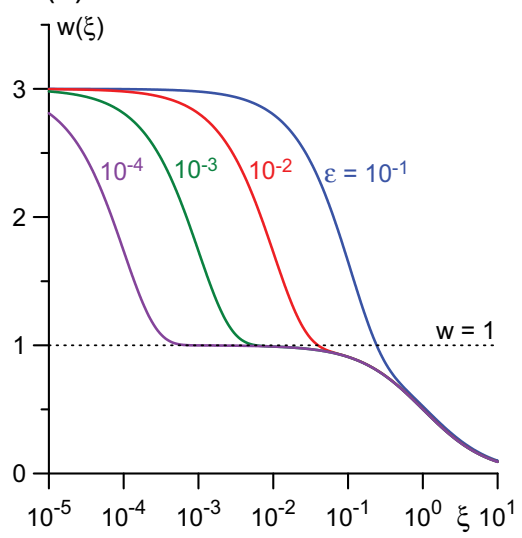

(b)

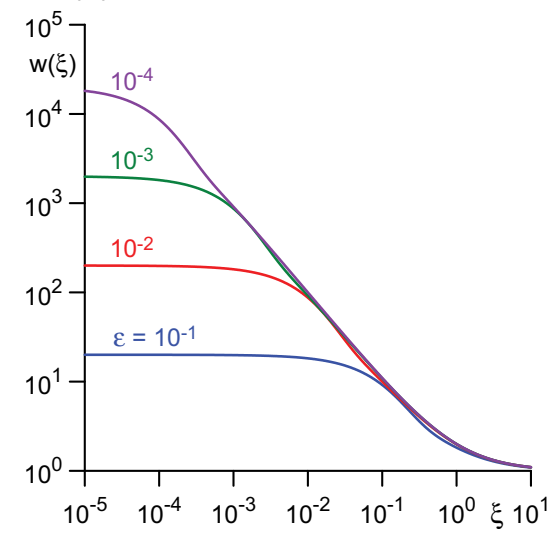

(c)

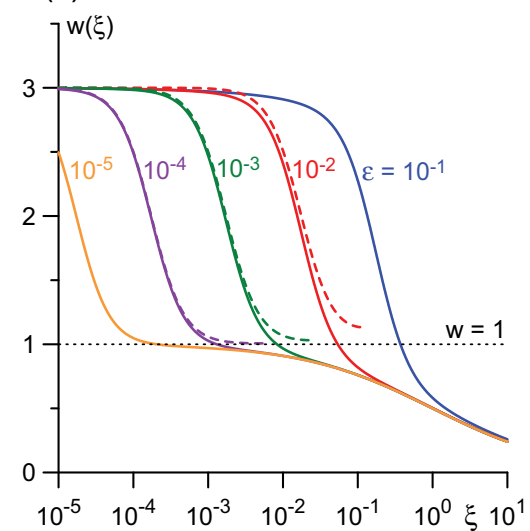

FIG. 1. Mathematical examples illustrating different scenarios of plasma-sheath transition. (a) Function (12). (b) Function (14). (c) Solid lines: Function (16). Dashed lines: Function (16) plus $\sqrt{\xi}$.

A major difference between these two examples is that while function (12) is of the same order of magnitude in the outer and inner regions, values of function (14) in the outer region are asymptotically small compared to those in the inner region. Indeed, functions $w^{(o)}$ and $w^{(i)}$ given by Eq. (13) are of the same order of magnitude (unity), while function $w^{(o)}$ given by Eq. (15), being of the order unity, is much smaller than $w^{(i)}$ given by the same equation, which is of the order $1 / \varepsilon$. As a consequence, asymptotic matching occurs in essentially different ways: on a constant (equal to 1 ) in the first example and on an algebraic function $1 / \xi$, which describes the increase of $w$ from order unity in the outer region to order $1 / \varepsilon$ in the inner region, in the second example. The latter difference is clearly seen in Figures 1(a) and 1(b): while $w(\xi)$ is virtually constant (and equal to 1 ) in the range $\varepsilon \ll \xi \ll 1$ for small $\varepsilon$ in Figure 1(a), in Figure 1(b) $w(\xi)$ is variable in the range $\varepsilon \ll \xi \ll 1$ and cannot be characterized by a number.

The third example is

$$
w(\xi)=\frac{1+\varepsilon}{1+\varepsilon+\sqrt{\xi}}+\frac{6 \varepsilon^{2}}{3 \varepsilon^{2}+\xi^{2}} .
$$

This function is plotted in Figure 1(c). The outer and inner approximations in this example are, respectively,

$$
w^{(o)}(\xi)=\frac{1}{1+\sqrt{\xi}}, \quad w^{(i)}(\eta)=1+\frac{6}{3+\eta^{2}} .
$$

Asymptotic matching occurs on a constant as in the first example, however the plateau in the range $\varepsilon \ll \xi \ll 1$ in Figure 1(c) appears for smaller values of $\varepsilon$ and is less pronounced than in Figure 1(a). One can assume for definiteness that a plateau is present if the reduction of function $w(\xi)$, say, from 1 to 0.7 requires an increase in $\xi$ by a factor of at least 10 . In other words, if $\xi_{1}$ and $\xi_{2}$ are the roots of equations $w\left(\xi_{1}\right)=1$ and $w\left(\xi_{2}\right)=0.7$, then the plateau is present provided that $\xi_{2} / \xi_{1} \geq 10$. Values of these roots for relevant $\varepsilon$ are given in Table I. One can see that the plateau appears in the first example for $\varepsilon \leq 10^{-2}$ and in the third example for $\varepsilon \leq 10^{-3}$.
The reason for this difference is that the outer and inner approximations tend to 1 in the intermediate region in the third example slower than in the first example. Indeed, the difference $w^{(o)}(\xi)-1$ for $\xi \rightarrow 0$ decays proportionally to $\xi$ in the first example and to $\sqrt{\xi}$ in the third example; the difference $w^{(i)}(\eta)-1$ for $\eta \rightarrow \infty$ decays exponentially in the first example and proportionally to $\eta^{-2}$ in the third example. As an illustration, also shown in Figure 1(c) is the function (16) for $\varepsilon=10^{-2}, 10^{-3}, 10^{-4}$ to which $\sqrt{\xi}$ has been added. One can see that the plateau for $\varepsilon=10^{-3}, 10^{-4}$ indeed becomes better pronounced.

\section{B. Possible scenarios of plasma-sheath transition}

Let us relate the above-described scenarios to the plasma-sheath transition near a negative solid surface (wall or cathode) in a weakly ionized plasma. To this end, we set $\xi=x / L$ and $\varepsilon=\lambda_{D} / L$, where $L=1$ in the case of a collisionless sheath and $L=\min \left(l_{i}, \Delta\right)$ in the cases of a moderately to strongly collisional sheath. In all the cases, $\varepsilon \ll 1$.

In these designations, the outer region in the examples of Sec. IV A corresponds to $x$ of the order of $L$ and represents the presheath. The inner region corresponds to $x$ of the order of $\lambda_{D}$ and represents the sheath. Figure 1 depicts two possible scenarios of variation of ion speed in the plasma-sheath transition, with $w$ representing $\left|v_{i}\right|$ normalized by a characteristic ion speed in the presheath. In the scenario depicted in Figures 1(a) and 1(c), the ion speed distribution reveals a

TABLE I. Appearance of plateau.

\begin{tabular}{lccccc}
\hline \hline Function & $\varepsilon$ & $\xi_{1}$ & $\xi_{2}$ & $\xi_{2} / \xi_{1}$ & Plateau \\
\hline$(12)$ & $10^{-1}$ & 0.241 & 0.502 & 2.08 & Absent \\
$(12)$ & $10^{-2}$ & 0.0397 & 0.433 & 10.9 & Present \\
$(16)$ & $10^{-2}$ & 0.0539 & 0.212 & 3.93 & Absent \\
$(16)$ & $10^{-3}$ & 0.00830 & 0.184 & 22.2 & Present \\
$\begin{array}{l}\text { Numerics, } p=0.1 \mathrm{~Pa}, \\
j_{i}=0.42 \mathrm{Am}^{-2}\end{array}$ & $10^{-2}$ & 0.0898 & 0.281 & 3.13 & Absent \\
$\begin{array}{l}\text { Numerics, } p=0.1 \mathrm{~Pa}, \\
j_{i}=42 \mathrm{Am}^{-2}\end{array}$ & $10^{-3}$ & 0.0134 & 0.175 & 13.1 & Present \\
\hline \hline
\end{tabular}


plateau in the intermediate region $\varepsilon \ll \xi \ll 1$ (or, equivalently, $\left.\lambda_{D} \ll x \ll L\right)$ and the smaller $\varepsilon$ is, the better this plateau is pronounced. The value corresponding to this plateau in the limit $\varepsilon \rightarrow 0$ has the meaning of a speed with which ions leave the presheath and enter the sheath; the Bohm speed. No such plateau exists in the scenario depicted in Figure 1(b), so the concept of a definite speed with which ions enter the sheath and, therefore, the Bohm criterion are meaningless. The first scenario occurs if the ion speed is of the same order of magnitude in the sheath and presheath. The second scenario occurs if the ion speed in the sheath is much higher than that in the presheath (for the function (14), which is shown in Figure 1(b), it is greater by a factor of the order of $\left.\varepsilon^{-1}=L / \lambda_{D}\right)$.

Thus, a simple way to find out what scenario of plasmasheath transition occurs in a particular situation and whether the Bohm criterion is meaningful is to compare ion velocities in the sheath and the presheath. This can be done as follows. If the negative solid surface being considered is an insulating wall, then the voltage drop in the space-charge sheath is of the order of $k T_{e} / e$. If the surface being considered is a cathode, then the voltage drop in the sheath may be much higher, however only the outer part of the near-cathode sheath where variation of potential is of the order of $k T_{e} / e$ is relevant as far as the plasma-sheath transition is concerned. Therefore, it is legitimate to assume that the sheath voltage is of the order of $k T_{e} / e$. The voltage drop in the presheath also is of the order of $k T_{e} / e$. In the case of a collisionless sheath, the ion speed in the sheath is of the order of $v_{s}$; since the presheath is collisionless or moderately collisional (its thickness is of the order of $\min \left(\lambda_{s}, l_{i}\right)$ in this case), the ion speed in the presheath is of the same order $v_{s}$. If the sheath is (moderately or strongly) collisional, $\lambda_{s} \lesssim \lambda_{D} \ll L=\min \left(l_{i}, \Delta\right)$, then the presheath is collision-dominated and the ion speed in the presheath is much smaller than that in the sheath; see asymptotic estimates in the Appendix for details. It follows that the scenario sketched in Figures 1(a) and 1(c) occurs and the Bohm criterion is meaningful if the sheath is collisionless; the scenario sketched in Figure 1(b) occurs and the Bohm criterion is meaningless if the sheath is moderately to strongly collisional.

It should be stressed that the above reasoning, while being simple, is not simplistic: the fact that a function having the same order of magnitude in adjacent asymptotic zones is to the first approximation constant on intermediate length scales represents the bottom mathematical meaning of the Bohm criterion.

Asymptotic estimates of the plasma-sheath transition for collisionless to moderately collisional to collision-dominated sheaths are given in the Appendix. These estimates conform, on the one hand, to the above reasoning and, on the other hand, to the results of the asymptotic analysis by means of the method of matched asymptotic expansions performed in Refs. 12 and 13 and 29-32 for the cases of collisionless and collision-dominated sheath, respectively. In particular, these estimates show that in the case of moderately to strongly collisional sheath the ion speed varies in the intermediate region $\lambda_{D} \ll x \ll L$ proportionally to $1 / x$, as in the example (14), if the ion-atom interaction is described with the use of the constant collision frequency model, and proportionally to $1 / \sqrt{x}$, if the model of constant ion mean free path is used.

The above considerations refer to the first-approximation solution. If the analysis for the case of a collisionless sheath is extended to the second approximation, then the intermediate transition layer must be considered as discussed in Sec. III. The asymptotic behavior of the second-approximation term $u_{i}$ in the transition layer for small and large $x / \lambda_{D}^{4 / 5} l^{1 / 5}$, cited at the end of Sec. III B, must coincide with the second term of, respectively, the expansion for large $\eta$ of the firstapproximation sheath solution and the expansion for small $\xi$ of the first-approximation plasma solution; the van Dyke asymptotic matching principle [Ref. 6, Eq. (5.24)]. Hence, the latter expansions are, respectively, $\left|v_{i}\right| \approx v_{s}\left(1+6 / \eta^{2}\right)$ and $\left|v_{i}\right| \approx v_{s}(1-\sqrt{\xi})$.

It follows that the example (16) and Figure 1(c) are more representative of a collisionless sheath than the example (12) and Figure 1(a). One should expect therefore that the plateau in the ion speed distribution manifesting the Bohm criterion is reasonably well pronounced only for $\varepsilon$ of the order $10^{-3}$ or smaller.

\section{Identifying the Bohm criterion in results of numerical calculations}

Let us consider as an example, a numerical solution of Eqs. (1)-(4) for the case of a region without ionization near a floating wall. The ion current density $j_{i}$ is considered as a control parameter, $T_{e}=3 \mathrm{eV}$, and $\nu_{i}=\left|v_{i}\right| / \lambda_{i}$, where $\lambda_{i}=\lambda_{i}\left(\left|v_{i}\right|\right)$ is the local ion-atom mean free path which is approximated for argon as $\lambda_{i}=\frac{1}{n_{a} \sigma_{0}} \sqrt{\frac{v_{i}^{2}}{v_{0}^{2}+v_{i}^{2}}}$ with $\sigma_{0}=10^{-18} \mathrm{~m}^{2}$ and $v_{0}=550 \mathrm{~ms}^{-1} \cdot{ }^{22}$ A boundary condition at the wall is $j_{i}=e n_{e} \sqrt{k T_{e} / 2 \pi m_{e}}$, two boundary conditions on the plasma side are those of quasi-neutrality and ion motion being drift, $v_{i}=e E / m_{i} \nu_{i}$.

The Bohm speed under these conditions is $v_{s}=2.7$ $\times 10^{3} \mathrm{~ms}^{-1}$. The characteristic ion mean free path $\lambda_{s}$ and the Debye length $\lambda_{D}$ are estimated as $\lambda_{s}=\lambda_{i}\left(v_{s}\right), \quad \lambda_{D}$ $=\left(\varepsilon_{0} k T_{e} v_{s} / e j_{i}\right)^{1 / 2}$; it will be seen that these lengths are characteristic of the sheath for all conditions considered here. In order to give reference values, we indicate that $\lambda_{s}=4.0 \mathrm{~mm}$ for the plasma pressure $p=1 \mathrm{~Pa}$ and $\lambda_{D}=0.41 \mathrm{~mm}$ for $j_{i}=0.42 \mathrm{Am}^{-2}$.

Distributions of ion speed in the near-wall region for several combinations $\left(p, j_{i}\right)$ are shown in Figures 2 and 3. Three circles on each curve represent points where the charge separation $\left(n_{i}-n_{e}\right) / n_{i}$ reaches, in the direction from the plasma to the wall, $10 \%, 20 \%$, and $50 \%$, respectively. In order to illustrate the character of the plasma-sheath transition, in each case the distribution should be plotted on the presheath scale. Variants shown in Figure 2 are chosen that the sheath be weakly collisional, $\lambda_{D} / \lambda_{s} \leq 10^{-1}$. In this case, the presheath is represented by the Knudsen layer and $L=\lambda_{s}$, hence $\varepsilon=\lambda_{D} / \lambda_{s}$. Since $\lambda_{s}$ varies over two orders of magnitude for the set of variants depicted in Figure 2, the distance $x$ is normalized by $\lambda_{s}$. Since $\left|v_{i}\right|$ in the presheath does not vary by orders of magnitude from one variant to the others, there is no need to normalize it. 


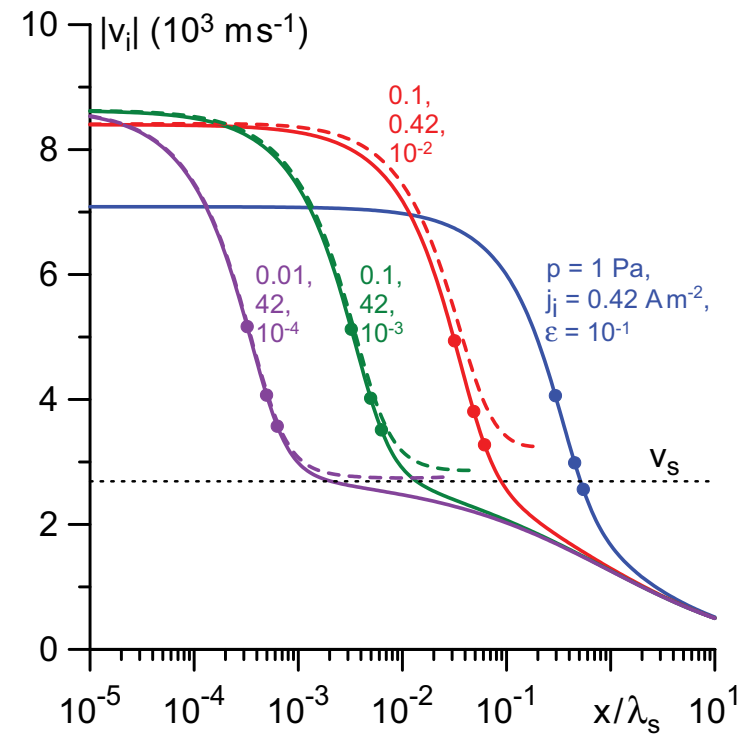

FIG. 2. Plasma-weakly collisional sheath transition. $\varepsilon=\lambda_{D} / \lambda_{s}$. Solid lines: Numerical solution. Dashed lines: Numerical solution plus $v_{s} \sqrt{x / \lambda_{s}}$.

As expected, Figure 2 is qualitatively similar to Figure 1(c). It is natural to identify the presence of a plateau at the Bohm speed by means of the same criterion as in Sec. IV A: the plateau is present if the reduction of the ion speed from $v_{s}$ to $0.7 v_{s}$ requires an increase in $x$ by a factor of at least 10 . Corresponding values of the normalized coordinate $\xi=x / \lambda_{s}$ are shown in Table I. One can see that the plateau appears for $\varepsilon \leq 10^{-3}$, similarly to what happens in the third example treated in Sec. IV A.

In order to make this asymptotic feature more distinct, one can modify the numerical solution similarly to how the function (16) was modified in Sec. IV A. Dashed lines in Figure 2 depict the numerical solution from which the function $-v_{s} \sqrt{x / \lambda_{s}}$, representing the second term of expansion in $x / \lambda_{s}$ of the (quasi-neutral) solution describing the presheath, was subtracted. As in Sec. IV A, the plateau for $\varepsilon=10^{-3}$, $10^{-4}$ becomes better pronounced.

Variants shown in Figure 3 are chosen so that the sheath be moderately collisional, $\lambda_{D}=\lambda_{s}$. In this case, the presheath is represented by the whole plasma slab and $L$ equals the slab width $\Delta$, hence $\varepsilon=\lambda_{D} / \Delta$. Let us assume for defi- niteness that $\Delta=4 \mathrm{~cm}$. One can see from Figure 3(a) that the ion speed in the sheath is of the order of $v_{s}$, hence the scales $\lambda_{D}$ and $\lambda_{s}$ evaluated in terms of $v_{s}$ are characteristic of the sheath, although not of the presheath. Also shown in Figure 3(a) is the ion speed evaluated by means of the drift approximation in terms of the local electric field. One can see that the drift approximation ensures a good accuracy in the presheath, which could be expected since the presheath in the cases of moderately to strongly collisional sheath is collision-dominated as discussed in Sec. IV B and in the Appendix.

One can see from Figure 3(a) that $\left|v_{i}\right|$ in the presheath varies by orders of magnitude from one variant to the others. Therefore, $v_{i}$ should be normalized if one wishes to analyze the character of the plasma-sheath transition. Since $\Delta$ is the same for all variants, there is no need to normalize $x$. Such representation is shown in Figure 3(b). As expected, this figure is qualitatively similar to Figure 1(b).

One can conclude that the pattern of plasma-sheath transition revealed by numerical calculations is precisely as predicted by the asymptotic reasoning. In particular, the numerical solutions reveal the Bohm criterion for small enough values of the ratio $\lambda_{D} / \lambda_{S}$, and "small enough" means the order of $10^{-3}$ or smaller.

Results of numerical solution of this problem for $p \geq 0.1 \mathrm{~Pa}$ and $j_{i}=0.42 \mathrm{Am}^{-2}$ are given in Ref. 22 . It is seen from Figure 2 that these conditions are not suitable for looking for the Bohm criterion; higher $j_{i}$ and/or lower $\mathrm{p}$ should be considered to this end. On the other hand, the line for $\mathrm{p}=0.1 \mathrm{~Pa}, j_{i}=0.42 \mathrm{Am}^{-2}$ in Figure 2 reveals a change in slope around the Bohm speed and this change represents the beginning of formation of the plateau manifesting the Bohm criterion.

\section{Discussion}

The classic Bohm criterion ${ }^{33}$ defines the speed with which cold ions enter a collisionless space-charge sheath from the adjacent quasi-neutral plasma. As Bohm himself put it: "the penetration [of the sheath electric field into the plasma] must be such as to accelerate ions to a velocity corresponding to half the mean electron kinetic energy." This definition is mathematically meaningful: there is a plateau in (a)

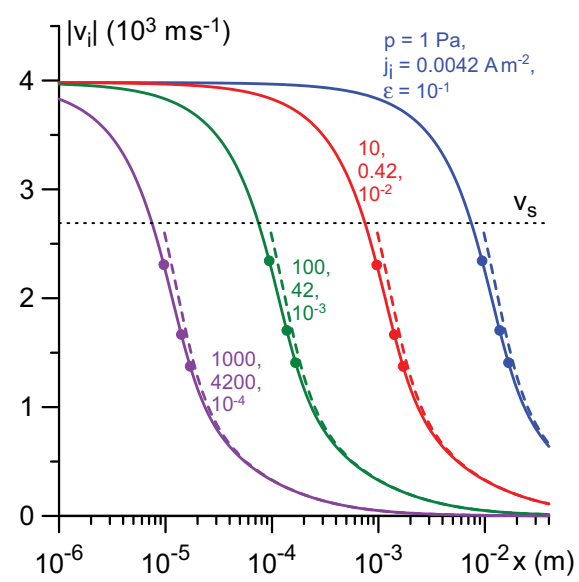

(b)

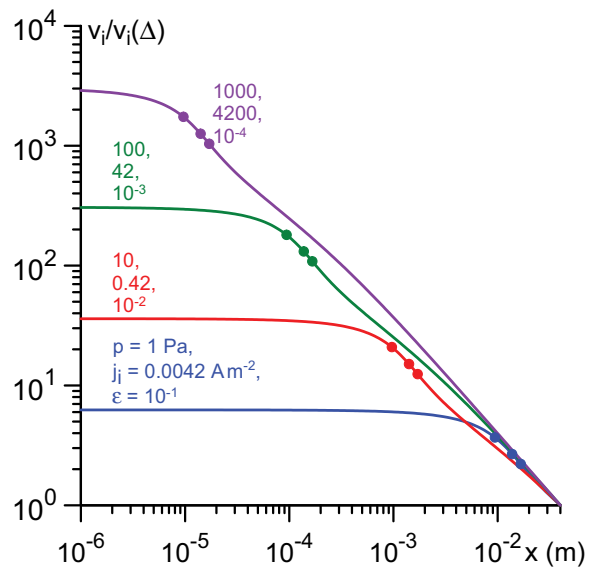

FIG. 3. Plasma-moderately collisional sheath transition. $\varepsilon=\lambda_{D} / \Delta$. Solid lines: Numerical solution. Dashed lines: Drift approximation. 
the ion speed distribution in the region $\lambda_{D} \ll x \ll L$ in the case of a collisionless sheath as illustrated by Figures 1(c) and 2 . The presheath scale $L$ in the case of a collisionless sheath represents the smallest of all relevant length scales excluding $\lambda_{D}$ but including the ion-atom mean free path $\lambda_{s}$. The plateau is pronounced the better, the smaller the ratio $\lambda_{D} / L$ is; or, in other words, the higher the degree of plasma quasi-neutrality is and the smaller effects which come into play on the presheath scale $L$, including ion collisions, are in the sheath; or, equivalently, the better pronounced the difference between the plasma and the sheath is. The plateau becomes ideal (horizontal) in the limiting case of infinitely small $\lambda_{D} / L$, where deviations from quasi-neutrality are neglected in the plasma and effects which come into play on the plasma scale $L$, including ion collisions, are neglected in the sheath. The Bohm criterion becomes exact in this ideal situation and the speed corresponding to the (horizontal) plateau is the Bohm speed. It is important to stress that this reasoning does not involve the concept of a sheath edge, i.e., a boundary separating the quasi-neutral plasma and the spacecharge sheath, which is meaningless in Bohm's model (although it is meaningful in the Child-Langmuir model of ion sheath; see discussion in Ref. 4).

There is no plateau in the ion speed distribution in the intermediate region $\lambda_{D} \ll x \ll L$ in the case of a moderately collisional to collision-dominated sheath as illustrated by Figures 1(b) and 3, and therefore no sense in talking of a definite speed with which ions enter the sheath. The presheath length scale $L$ in this case represents the smallest of all relevant length scales excluding $\lambda_{D}$ and $\lambda_{s}$.

The terms collisionless, or moderately collisional, or collision-dominated sheath in the asymptotic treatment mean that the ratio $\lambda_{D} / \lambda_{s}$ is considered as a small parameter, or the lengths $\lambda_{D}$ and $\lambda_{s}$ are considered as comparable, or the ratio $\lambda_{S} / \lambda_{D}$ is considered as a small parameter. For practical purposes, a numerical estimate is useful of how small the ratio $\lambda_{D} / \lambda_{s}$ should be for the Bohm criterion to be applicable. If one assumes for definiteness that a plateau in the ion speed distribution is reasonably well pronounced provided that a reduction of the ion speed occurring over an order-of-magnitude increase in $x$ does not exceed $30 \%$, then $\lambda_{D} / \lambda_{s}$ should be of the order of $10^{-3}$ or smaller.

The smallness of the latter value reveals a severe restriction on practical applications of the Bohm criterion, therefore attempts to define a collisionally modified Bohm criterion are understandable. However, such definitions do not represent a Bohm criterion in Bohm's sense, simply because the concept of a definite speed with which cold ions enter the sheath, while being meaningful for collisionless sheaths treated by Bohm, becomes meaningless as collisions come into play. In other words, Bohm's definition has no analogue for collisional sheaths and one cannot introduce a collisionally modified criterion except using one's own definition of the sheath edge (like the point where the electric field takes a specific value, or the inflection point of the spatial distribution of the ion or electron density, or the point of a removable singularity appearing after a certain transformation of governing equations, etc.), which is inevitably arbitrary. Without questioning the practical usefulness of such- type models, one should recognize that the term "Bohm criterion," when applied to collisional sheaths, is likely to contribute to further confusion, as has always happened in the past, and better be avoided.

\section{CONCLUSIONS}

A transformation of the ion momentum equation renders unnecessary resorting to the third approximation in the theory of intermediate transition layer between a quasi-neutral plasma and a collisionless sheath and thus clearly reveals the physics involved. Since the transition layer is positioned between the plasma and the sheath, ion-atom collisions and/ or ionization are no longer significant there while separation of charges is not significant yet, so the ion motion is dominated by inertia and the electrostatic force. However, the latter forces virtually cancel in the vicinity of the sonic barrier and both above-mentioned weak effects (ion-atom collisions and/or ionization and separation of charges) also play a role. It seems that the term "transonic layer," suggested in Ref. 14, more adequately reflects the physics than the conventionally used terms intermediate or transition layer.

The Bohm criterion represents a manifestation of one of general scenarios of asymptotic matching, namely, matching on a constant, and is not directly related to the sheath edge singularity. In order to show it, the relevant scenarios are illustrated by simple mathematical examples and occurrence of these scenarios in plasma-sheath transition is analyzed by means of asymptotic reasoning and numerical calculations. In the case of a collisionless sheath, there is a plateau in the ion speed distribution in the intermediate region between the sheath and the presheath. (The distribution should be plotted on a logarithmic scale, as usual in multi-scale problems.) The value corresponding to this plateau has the meaning of a speed with which ions leave the presheath and enter the sheath; the Bohm speed. There is no such plateau in the cases of a moderately collisional to collision-dominated sheath, and hence no sense in talking of a speed with which ions enter the sheath.

For the Bohm criterion to be applicable or, in other words, for the plateau in the ion speed distribution to be pronounced reasonably well, the ratio $\lambda_{D} / \lambda_{s}$ should be small enough. If one assumes for definiteness that "pronounced reasonably well" means a reduction of the ion speed by no more than $30 \%$ over an order-of-magnitude increase in $x$, then " $\lambda_{D} / \lambda_{s}$ small enough" means of the order of $10^{-3}$ or smaller.

Since the effect of collisions causes the plateau in the ion speed distribution to disappear, any collisionally modified Bohm criterion is not a Bohm criterion in Bohm's sense: it does not define a speed with which ions enter the sheath, simply because the concept of a definite speed with which cold ions enter the sheath, while being meaningful for collisionless sheaths, is meaningless when collisions come into play. In other words, Bohm's definition has no analogue for collisional sheaths, hence one cannot introduce a collisionally modified criterion except using one's own definition. This explains why the collisionally modified Bohm criteria available in the literature are different. 
The asymptotic reasoning and estimates employed in this work are simple. However, they are not simplistic, in contrast to what someone without experience with the method of matched asymptotic expansions could think: a treatment with the use of formal asymptotic expansions confirms these reasoning and estimates, by showing that they result in a self-consistent solution, but does not add to their essence.

\section{ACKNOWLEDGMENTS}

This work was supported by FCT - Fundação para a Ciência e a Tecnologia of Portugal through projects PTDC/ FIS/68609/2006 and PEst-OE/MAT/UI0219/2011. M.S.B. gratefully acknowledges stimulating discussions with R. N. Franklin.

\section{APPENDIX: PLASMA-SHEATH TRANSITION FOR COLLISIONLESS TO COLLISION-DOMINATED SHEATHS VIA ASYMPTOTIC ESTIMATES}

\section{Ion velocities in collision-free to collision- dominated sheath and presheath}

As discussed in Sec. IV B, the sheath voltage drop may be assumed to be of the order of $k T_{e} / e$. The electric field $E$ in the sheath is of the order of $k T_{e} / e \lambda_{D}$. The order of magnitude of ion speed in the sheath depends on the relationship between $\lambda_{D}$ and $\lambda_{s}$. If $\lambda_{s} \gtrsim \lambda_{D}$, i.e., motion of the ions in the sheath is collision-free or moderately collisional, then $v_{i}$ is of the order of $v_{s}=\sqrt{k T_{e} / m_{i}}$. If $\lambda_{s} \ll \lambda_{D}$, i.e., ion motion in the sheath is dominated by collisions, then the regime of this motion depends on the relation between work of the electric field over an ion mean free path, $e E \lambda_{s}$, and the mean thermal energy of the neutral particles, $k T_{a}$; e.g., Ref. 34. Estimates of this work are restricted to the case of a high-electric field regime, which occurs if $e E \lambda_{s} \gg k T_{a}$, or, equivalently, $\lambda_{s} / \lambda_{D} \gg T_{a} / T_{e}$. (Since $T_{a} / T_{e}$ is typically of the order of $10^{-2}$ under conditions of a glow discharge, this case is representative.) In this case, $v_{i}$ is of the order of $\sqrt{e E \lambda_{s} / m_{i}}$ or, equivalently, of the order of $v_{s} \sqrt{\lambda_{s} / \lambda_{D}}$.

Since the voltage drop in the presheath also is of the order of $k T_{e} / e$, the above estimates are valid also for the presheath provided that $\lambda_{D}$ is replaced with $L$.

\section{Transition to a collision-free sheath}

If the ion motion in the sheath is collision-free, then the ion motion in the presheath can be either collision-free or moderately collisional. [But it cannot be collision-dominated: a collision-dominated bulk plasma and a collisionfree sheath are separated by a moderately collisional region (a Knudsen layer) and it is the Knudsen layer that represents the presheath in such situation.] In other words, the hierarchy of length scales in this case is $\lambda_{D} \ll L \lesssim \lambda_{s}$. If the column of a low-pressure glow discharge is considered as an example, then the presheath is represented by the plasma column if the column is collisionless or moderately collisional and by the Knudsen layer if the column is collision- dominated: $L=R$ if $\lambda_{s} \gtrsim R$ and $L=\lambda_{s}$ if $\lambda_{s} \ll R$ (here $R$ is radius of the discharge tube). Note that the ionization length in this example is comparable to $R$ and need not be considered separately.

The ion velocities in the sheath and presheath are of the same order $v_{s}$. Hence, the scenario depicted in Figures 1(a) and 1(c) occurs. This is the classic case treated by Bohm ${ }^{33}$ on intuitive grounds and by subsequent workers ${ }^{12,13}$ by means of the method of matched asymptotic expansions. There are also many further works taking into account finite ion temperature, multiple ion species, etc.

\section{Transition to a collision-dominated sheath}

If the ion motion in the sheath is dominated by collisions, then the hierarchy of length scales is $\lambda_{s} \ll \lambda_{D} \ll L$ and the ion motion in the presheath is collision-dominated as well. In the example of a low-pressure glow discharge column, the presheath is represented by the (collision-dominated) plasma column: $L=R$.

Two models of ion-atom interaction are widely used in plasma-sheath problems: the model of Maxwell molecules (constant collision frequency) and the model of rigid spheres (constant ion mean free path). In the framework of the model of constant collision frequency, the ion mobility does not depend on electric field and $v_{i}$ is proportional to $E$. The ratio of ion speed in the sheath to that in the presheath is of the same order as the ratio of the corresponding electric fields, i.e., of the order of $L / \lambda_{D}$, and much larger than unity. Hence, the scenario depicted in Figure 1(b) occurs. This case was treated in Refs. 29-31; one should mention also works, ${ }^{35,36}$ where the case of a high-voltage sheath was considered. No accurate intuitive theory, similar to Bohm's theory for the case of collisionless sheath, was given for this case.

Note that while comparing the estimates of this work with those resulting from analysis, ${ }^{29-31}$ one should keep in mind the following. Since the ion current is of the same order of magnitude in the sheath and presheath, the different orders of ion velocities in the sheath and presheath are associated with different orders of the charged-particle density: the density in the sheath is smaller by a factor of $\lambda_{D} / L$. In this work, $\lambda_{D}$ designates the Debye length estimated in terms of charged particle density characteristic for the sheath. In Refs. 29-31, the Debye length is estimated in terms of charged particle density characteristic for the presheath. Let us designate the latter length by $\tilde{\lambda}_{D}$. The two Debye lengths are related by $\tilde{\lambda}_{D} / \lambda_{D}=\sqrt{\lambda_{D} / L}$. It follows that $\lambda_{D}=\tilde{\lambda}_{D}^{2 / 3} L^{1 / 3}$. Hence, the scale of the sheath may be expressed as $\tilde{\lambda}_{D}^{2 / 3} L^{1 / 3}$, in agreement with the asymptotic results. ${ }^{29-31}$

In the framework of the model of constant ion mean free path, $\left|v_{i}\right|$ is proportional to $\sqrt{|E|}$ and the ratio of ion speed in the sheath to that in the presheath is of the order of $\sqrt{L / \lambda_{D}}$, i.e., much larger than unity. It follows that the scenario depicted in Figure 1(b) occurs again. It follows also that the ion speed varies in the intermediate region $\lambda_{D} \ll x \ll L$ proportionally to $1 / \sqrt{x}$, rather than to $1 / x$ as in the constant collision frequency model and in the example (14). However, 
this difference does not change the pattern. One finds $\tilde{\lambda}_{D} / \lambda_{D} \overline{\overline{4 / 5}}\left(\lambda_{D} / L\right)^{1 / 4}$ in this case and the sheath scale is $\lambda_{D}=\tilde{\lambda}_{D}^{4 / 5} L^{1 / 5}$, in agreement with the asymptotic results. ${ }^{32}$

\section{Transition to a moderately collisional sheath}

If the ion motion in the sheath is moderately collisional, then the hierarchy of length scales is $\lambda_{s} \sim \lambda_{D} \ll L$ and the ion motion in the presheath is collision-dominated. In the example of a low-pressure glow discharge column, the presheath is again represented by the (collision-dominated) plasma column: $L=R$.

The ion speed is of the order of $v_{s}$ in the sheath and of the order of $v_{s} \sqrt{\lambda_{s} / L}$, i.e., much smaller, in the presheath. The scenario depicted in Figure 1(b) occurs.

In the framework of the model of constant ion mean free path, the ion mean free path in the presheath is the same as that in the sheath. Since the latter is comparable to $\lambda_{D}$, the ratio of ion speed in the sheath to that in the presheath is of the order of $\sqrt{L / \lambda_{D}}$ and $\left|v_{i}\right|$ varies in the intermediate region $\lambda_{D} \ll x \ll L$ proportionally to $1 / \sqrt{x}$. In the framework of the model of constant collision frequency, $\lambda_{i}$ is proportional to $\left|v_{i}\right|$. One finds that the ratio of ion speed in the sheath to that in the presheath is of the order of $L / \lambda_{D}$ and the ion speed varies in the intermediate region $\lambda_{D} \ll x \ll L$ proportionally to $1 / x$.

In summary, the plasma-sheath transitions in the cases of collision-dominated and moderately collisional sheaths follow similar patterns. Of course, this should have been expected since the intermediate region is collisiondominated in both cases.

\footnotetext{
${ }^{1}$ K.-U. Riemann, J. Phys. D: Appl. Phys. 24(4), 493 (1991).

${ }^{2}$ R. N. Franklin, J. Phys. D: Appl. Phys. 36(22), R309 (2003).

${ }^{3}$ J. E. Allen, Plasma Sources Sci. Technol. 18(1), 014004 (2009).

${ }^{4}$ M. S. Benilov, Plasma Sources Sci. Technol. 18(1), 014005 (2009).

${ }^{5}$ K.-U. Riemann, Plasma Sources Sci. Technol. 18(1), 014006 (2009).
}

${ }^{6}$ M. van Dyke, Perturbation Methods in Fluid Mechanics (Parabolic, Stanford, CA, 1975).

${ }^{7}$ J. D. Cole, Perturbation Methods in Applied Mathematics (Blaisdell, Waltham, 1968).

${ }^{8}$ A. H. Nayfeh, Perturbation Methods (Wiley, New York, 1973).

${ }^{9}$ A. H. Nayfeh, Introduction to Perturbation Techniques (Wiley, New York, 1981).

${ }^{10}$ J. Kevorkian and J. D. Cole, Perturbation Methods in Applied Mathematics (Springer-Verlag, New York, 1981).

${ }^{11}$ A. H. Nayfeh, Problems in Perturbation (Wiley, New York, 1985).

${ }^{12}$ S. H. Lam, Phys. Fluids 8(1), 73 (1965).

${ }^{13}$ R. N. Franklin and J. R. Ockendon, J. Plasma Phys. 4(2), 371 (1970).

${ }^{14} \mathrm{~S}$. H. Lam, in Proceedings of VIIIth International Conference on Phenomena in Ionized Gases (IAEA, Vienna, 1967), Vol. I, pp. 545-568.

${ }^{15}$ K.-U. Riemann, Phys. Plasmas 4(11), 4158 (1997).

${ }^{16}$ I. D. Kaganovich, Phys. Plasmas 9(11), 4788 (2002).

${ }^{17}$ K.-U. Riemann, J. Seebacher, D. D. Tskhakaya, Sr., and S. Kuhn, Plasma Phys. Controlled Fusion 47(11), 1949 (2005).

${ }^{18}$ M. S. Benilov, Phys. Plasmas 7(1), 135 (2000)

${ }^{19}$ V. A. Godyak, Phys. Lett. A 89(2), 80 (1982).

${ }^{20}$ M. Khoramabadi, H. Ghomi, and M. Ghorannevis, J. Fusion Energy 29, 365 (2010).

${ }^{21}$ S. F. Masoudi and Z. Ebrahiminejad, Eur. Phys. J. D 59, 421 (2010).

${ }^{22}$ R. P. Brinkmann, J. Phys. D: Appl. Phys. 44(4), 042002 (2011).

${ }^{23}$ R. P. Brinkmann, in Abstracts 2011 IEEE International Conference Plasma Science (June 2011), p. 1, http://dx.doi.org/10.1109/PLASMA.2011.5992977.

${ }^{24}$ M. El Kaouini, H. Chatei, I. Driouch, and M. E. Hammouti, J. Fusion Energy 30, 199 (2011).

${ }^{25}$ X. Zou, H.-P. Liu, M.-H. Qiu, and X.-H. Sun, Chin. Phys. Lett. 28(12), 125201 (2011).

${ }^{26}$ R. N. Franklin, J. Phys. D: Appl. Phys. 44(15), 158001 (2011).

${ }^{27}$ R. P. Brinkmann, J. Phys. D: Appl. Phys. 44(15), 158002 (2011).

${ }^{28}$ R. N. Franklin, J. Phys. D: Appl. Phys. 36(22), 2821 (2003).

${ }^{29}$ I. M. Cohen, Phys. Fluids 6(10), 1492 (1963).

${ }^{30}$ J. L. Blank, Phys. Fluids 11(8), 1686 (1968).

${ }^{31}$ M. S. Benilov, Fluid Dyn. 17(5), 773 (1982).

${ }^{32}$ M. S. Benilov and R. N. Franklin, J. Plasma Phys. 67(2-3), 163 (2002).

${ }^{33} \mathrm{D}$. Bohm, in The Characteristics of Electrical Discharges in Magnetic Fields, edited by A. Guthrie and R. K. Wakerling (McGraw-Hill, New York, 1949), pp. 77-86.

${ }^{34}$ E. A. Mason and E. W. McDaniel, Transport Properties of Ions in Gases (Wiley, New York, 1988).

${ }^{35}$ C. H. Su and S. H. Lam, Phys. Fluids 6(10), 1479 (1963).

${ }^{36}$ W. B. Bush and F. E. Fendell, J. Plasma Phys. 4(2), 317 (1970). 